\title{
BIOMIMETIC TACTILE SENSING
}

\author{
Ravinder Dahiya ${ }^{1}$, Calogero Oddo ${ }^{2}$, Alberto Mazzoni ${ }^{2}$, Henrik Jörntell ${ }^{3}$ \\ ${ }^{I}$ Electronics and Nanoscale Engineering, University of Glasgow, UK, ${ }^{2}$ The Biorobotics Institute, Scuola Superiore Sant 'Anna, Pisa, \\ Italy. ${ }^{3}$ Department of Medical Science, Lund University, Sweden.
}

Tactile sensing is an essential component in many applications such as robotics, haptics, rehabilitation, electrotextiles, prosthetics and many more. In the light of greater focus on man-machine interaction, the importance of artificial tactile sensing as an important feedback tool is also growing. Starting with an overview of tactile sensing, in humans and robots, this chapter presents artificial tactile sensing inspired from the humans sense of touch.

\section{INTRODUCTION}

What would happen if we had all sense modalities but the 'touch'? A simple experiment of manipulating objects after putting hands on an ice block for a moment can probably provide an answer to this question. In one such experiment, the skin on volunteers' hand was anesthetized so that tactile information from mechanoreceptors - the specialized nerve endings that respond to mechanical stimulation - was no longer available to the brain [3]. It was observed that even though volunteers' could see what they were doing, they could no longer maintain a stable grasp of the objects. This indicates that the movements become inaccurate and unstable in the absence of 'sense of touch'. The difficulties that humans could face in absence of sense of touch also point towards the importance of touch sense modality in robots, especially when they are expected to work in a human environment.

The touch sensing allows us to assess the size, shape, softness and texture of objects. It helps us understand the interaction behaviours of the real world objects - which depend on their weight; stiffness; on how their surface feels when touched; how they deform on contact and how they move when pushed. In applications such as robotics, tactile information is useful in a number of ways. In manipulative tasks, the tactile data is used as a control parameter and the tactile information typically includes contact point estimation, surface normal and curvature measurement and slip detection [7, 8]. A measure of the contact forces (both magnitude and direction) allows the grasp force control - needed to maintain stable grasps. In a real world interaction that involves, both, manipulative and exploratory tasks, the tactile information such as hardness/softness [9], temperature, vibrations etc. are needed to understand diverse properties of the contacted objects.

The need for suitable tactile sensing system in robotics has resulted in a large number of touch sensors and tactile sensing arrays by exploring nearly all modes of transduction viz: resistive, capacitive, piezoelectric, magnetic, quantum tunnelling, etc. [2, 10, 11]. Currently many research groups are also working towards developing skin-like artificial systems for large area tactile sensing. However, the touch sensors technology developed so far is largely insufficient for robotics, even if there is significant success in other areas such as mobile telephony. This could be attributed to number of factors such as availability of less than satisfactory sensory skins, insufficient methods of processing tactile data, the lack of systems approach and the lack mechanical flexibility and robust sensory structures. Often tactile data are processed with techniques adapted from visual data processing, which may not be a correct approach as the touch sensing is distributed over a much broader area than vision. As a consequence, the dense integration of sensors distributed in large and curved bodies is still lagging behind. This also presents the case for the implementing higher order cognitive functions for tactile action and perception. 


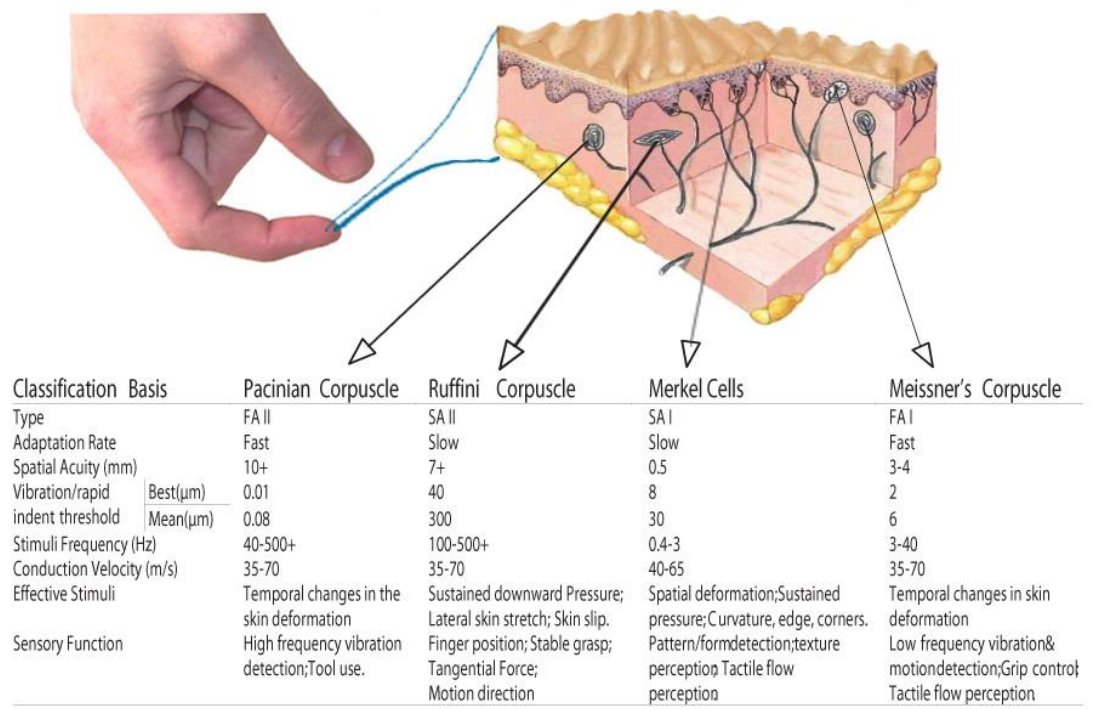

Figure 1. The classification, functions and location of various mechanoreceptors present in human glabrous skin [2]. Recent studies point towards the presence of Ruffini afferents in the hairy skin of human hand and not in the glabrous skin $[5,6]$.

Design of a meaningful tactile sensing system must be guided by a broad, but integrated, knowledge of how tactile information is encoded and transmitted at various stages. In this context, evolving biological systems could provide an answer. For centuries, biological systems have inspired engineers and scientists [12] and for tactile sensing the human touch sensing could be a good starting point. The human inspiration is also important in the absence of any comprehensive artificial tactile-sensing theory as the studies on human touch (e.g. neurophysiology of touch, skin biomechanics, movements for optimum exploration of material properties, object recognition, active and passive perception, selective attention etc.) could help in specifying important parameters like sensor density, resolution, location, bandwidth, etc. Starting with a brief discussion on the human touch sensing, this chapter presents the development artificial tactile sensing arrays that are inspired from touch sensing in humans. System level aspects such as wiring and information processing are discussed with final attention to neuromorphic signalling and the emulation of the neuronal organization and of the human somatosensory system from the periphery to the cortex. As this book focuses on nature inspired technologies, the discussion on the human sense of touch is brief and limited to the points of inspiration. For deeper understanding on human sense of touch, one may refer to the relevant literature [13-15].

\section{HUMAN SENSE OF TOUCH}

The touch sensing in humans comprises two main submodalities, i.e., "cutaneous" and "kinesthetic", or "exteroceptive" and "proprioceptive". The exteroceptive sense is based primarily on receptors with a cutaneous location and provides awareness of the stimulation of the outer surface of body. The proprioceptive or the kinesthetic sense has traditionally been ascribed to sensory receptors located within muscles, tendons, and joints [16] and provides information about the static and dynamic body postures (relative positioning of the head, torso, limbs, and end effectors). However, it is known that the sensors in the skin also play an important role in proprioception or kinesthetic sensation [17]. The term "tactile sensing", which is discussed in this chapter, is primarily based on the exteroceptive sense of the glabrous skin at the fingertips and the palm. 
Our ability to deal with the spatiotemporal perception of external stimuli, to discriminate among surface textures, temperature and to sense incipient slip and roll an object between fingers without dropping it etc. can be attributed to the specialized receptors embedded in the skin $[13,15]$. The receptors that are sensitive to pressure/vibration stimuli are termed 'mechanoreceptors' and those sensitive to pain/damage are called 'nocioceptors'. The response to thermal stimulus is believed to be mediated by separate "warm" and "cold" thermoreceptor population in the skin. The mechanoreceptors are the peripheral ends of neurons [18], which have their cell bodies located just outside the spinal cord, and the events generated at the mechanoreceptors are hence directly transferred into the central nervous system (CNS). The signal is transferred to the CNS via the neuron's axon, which for exteroceptive and proprioceptive sensors are denoted 'primary afferent'.

The receptors are distributed across the entire body with variable density. As an example, the number of mechanoreceptors, per square centimetre area, is estimated to be about 240 in the fingertips and about 60 in the palm of adult humans [19]. The spatial acuity is highest at fingertips, face and toes and lowest at thigh, shoulders and belly. Similarly, the receptors also have different receptive fields - the extent of body area to which a receptor responds - and different rates of adaptation. A fast-adapting (FA) receptor responds with bursts of action potentials when its preferred stimulus is first applied and when it is removed. In contrast, a slow-adapting (SA) receptor remains active throughout the period during which the stimulus is in contact with its receptive field. Thus, spatial-temporal limits and sensitivities of the receptors vary significantly across various body parts. The classification, functions, and location of various mechanoreceptors are given in Figure 1.

The moment the skin is stimulated, a variety of mechanical and neural events occur. On contact with an object, the skin conforms to its surface, which may if the object is compressible. The resulting skin deformation elicits mechanical strain patterns in the skin that may differ depending on the distance to the actual contact point, the edges of the contact point but also depending on the local biomechanical properties of the skin and the underlying anatomy of the fingertip, for example. These varied strain patterns are sensed by the local population of mechanoreceptors, which transforms the local strain pattern into an analogous electrical signal inside the neuron. Each mechanoreceptor thus represents a small portion of the skin-object interaction and encodes the spatiotemporal tactile information as spikes of action potentials-voltage pulses generated when the analogous voltage signal generated in the mechanoreceptor is greater than the threshold of the neuron. The amplitude of the stimulus is then transformed to a train of action potentials, which reflect the intensity and temporal profile of the mechanoreceptor events. Via the primary afferent fiber, the contact event related information is transmitted to the CNS for higher level processing and interpretation. The first stage in which this occurs is the cuneate nucleus in the lower part of the brainstem. The cuneate nucleus is essentially a mono-layer network with local inhibitory interneurons. From the cuneate nucleus, the information is transferred to the thalamus, another monolayer network with local inhibition and recurrent input from the neocortex, and from the thalamus to the primary sensory cortex.

An interesting feature of touch sensing in humans, which is also very useful for artificial tactile sensing, is the processing of tactile information at various stages of data transfer - thus reducing the computational burden of CNS. The processing of tactile data starts right from the peripheral level, i.e. already the local biomechanical properties and the exact forces applied will determine the patterns of skin strain induced by a certain skin-object interaction. As the local strain patterns are effectively transduced in the mechanoreceptors and conducted to the CNS [20], the spikes in the primary afferents already contain information that can be used to distinguish, for example, the curvature and the direction of force in the terminal phalanx [21]. A 
hypothetical model of human tactile processing, based on coincidence detection of neural events (Figure 2), was recently presented in [22]. This model proposes that: $(i)$ the relative timing of neural spikes elicited in (neighbouring) tactile units of the fingertip conveys significant information during manipulation activities; (ii) the spikes pass through neural afferents showing differentiated delays one to the other (due to dispersion of conduction velocity) in the pathways up to the second order (cuneate) neurons; (iii) second order neurons propagate the firing events to the higher stage in case that the differential delay introduced by the afferent pathways compensates the relative spike timing at the level of mechanoreceptors in the fingerpad; (iv) the tactile stimulus is pre-perceptually represented through the pattern of second order neurons being activated (i.e. those detecting a coincidence of incoming neural spikes, and thus propagating the firing up to the higher stage) during finger-surface mechanical interaction. Support for a modified version of this hypothesis, where the overall degree of correlation in afferent spike trains were considered rather than the first spike, was recently proposed as an explanation for input feature segregation in the cuneate nucleus [23].

The implications of the above discussion on the development of artificial tactile sensing systems are [11]:

- The presence of varied and distributed receptors with sharp division of functions, which calls for different types of miniaturized sensors - each optimally transducing a particular contact parameter.

- It is desirable to have multifunctional sensors that encode more than one contact parameter e.g. contact force and hardness detection by a sensor.

- The spatial density of the tactile sensors, distributed or arranged in an array, should be based on the body site or the target application site. For fingertips like sites, it should be about $1 \mathrm{~mm}$-which translates to an approximately $15 \times 10$ sensing element grids on a fingertip sized area.

- The sensors should demonstrate high sensitivity and wide dynamic range. Considering involvement of touch sensors in various exploratory tasks, a contact force sensitivity range of 1-1000 g wt. and a dynamic range of 1000:1 are desirable.

- The touch sensors could encode both magnitude and the direction of contact force. The response of tactile sensors distributed in an area could be used to obtain the direction of contact force as done by population mechanoreceptors in humans.

- Touch sensing elements should detect and encode both static and dynamic contact events. It is desirable to have tactile sensors that can detect vibrations up to $1 \mathrm{kHz}$.

- In humans, the tactile data is not directly conveyed to the brain. Instead, some processing takes place at



Figure 2. Hypothetical model, accounted by Johansson and Flanagan (2009), based on coincidence detection of neural events for the fast processing of afferent information. 
various stages of data transfer - perhaps to fit the limited throughput of the nervous system [22]. The tactile arrays or modules, with some level of preprocessing (data selection, local computation, etc.) at the sensory location, can be helpful in reducing the amount of information transfer to the central processing unit. According to this biologically-grounded model, computation also occurs along the wires that conduct information from artificial receptors to the central computational units.

\section{BIOMIMETIC ARTIFICIAL TOUCH}

Biorobotics fosters the convergence of technological achievements with new scientific knowledge and helps us understand the underlying natural phenomena and behaviours [24]. This approach has often been depicted in the light of bioinspiration and biomimetics, and it gives rise to morphological computation, a novel paradigm asserting the role of materials in taking over some of the processes normally attributed to control $[25,26]$, in conjunction with neuromorphic engineering in case of mimicry of neural mechanisms and architectures [27]. The organization of the somatosensory system presented in Section Error! Reference source not found. and illustrated in Figure 2 represents this understanding with fertile synergies between science and engineering. According to such model, the structure and shape of soft-tissues of the skin, the nonregular spatial distribution in array of mechanoreceptors, and the different conduction velocities along the afferent neural pathways up to the second-order cuneate neural stage have been hypothesised to implement computational operations on tactile information at pre-cortical stages [22]. This means that the skin, the mechanoreceptors and the afferent neural pathways are not just a mechanical interface medium, sensors and wires, respectively, but they implement computation thanks to their morphological characteristics. Particularly, neural pathways are not the impairment as wires are considered in traditional robotics. Their structure implements processing functions which would have required very complex sequential structures if instantiated centrally at brain level. Artificial touch can therefore show levels of biomimetism with respect to various characteristics, such as:

- the soft artificial skin that mediates the mechanical interaction with the tactile stimuli;

- the mechanotransduction core technology that converts mechanical stimulation to information;

- the neuromorphic representation of tactile information that allows the emulation of and integration with natural neuronal pathways.

These forms of biomimetism are briefly discussed hereafter with respect to current state of the art.

\subsection{Soft artificial skin}

Human tactile transduction is a complex energy conversion mechanism involving populations of mechanosensitive afferent fibres innervating the distal fingerpad and the skin with its different layers including fingerprints [28-31]. In the artificial emulation of a tactile sense, soft materials play a crucial role for potential future deployment in domains such as hand prosthetics: indeed, soft materials can increase the size of the contact area, thanks to their higher conformability, increase the contact friction coefficient (and thus the grasp stability), protect distributed embedded sensors which also provide better contact information, improve cosmetics, the latter being a relevant feature to enhance the final acceptability by the end user [32].

Biomimetics in the design of artificial fingers can go beyond the use of soft materials. Indeed, the effectiveness of employing soft materials is enhanced if anatomy and physiology of human fingers are considered [33]. The soft and pulpy tissue that is present between the skeletal bone and the skin addresses several functions, such as dissipating mechanical energy during impacts and protecting the bone tissues from 
lesions; because of its softness and of the elastic nature of the skin, the pulpy tissue can conform to most uneven surfaces of commonly used objects; further, due to its viscoelastic nature, it dissipates strain energy that is induced during manipulation of rigid objects, thus stabilizing the interaction [34, 35]. Similarly, microstructures such as fingerprints help in stable grasping of the objects, prevent them from slipping, and are also reported to help in identifying the roughness or smoothness of various surfaces $[28,36,37]$. Therefore the fabrication of soft robotic fingers, possibly with microstructures such as fingerprints, is important for a safer, more stable and reliant interaction with handled objects [38]. The packaging used in sensors for synthetic skin is mainly based on polymeric materials, such as silicone elastomers (e.g., polydimethylsiloxane in Dow Corning Sylgard 184® PDMS, polyorganosiloxanes and silica in Smooth-on DragonSkin ${ }^{\mathrm{TM}}$ and Ecoflex(@) and polyurethane rubbers (e.g., toluene diisocyanate and polyols in Polytek® Poly-74 Series) [30, 39]. These polymeric materials show compliant nature, resistance to temperature changes, mechanical toughness and the possibility to be healed. Also, the tensile strength and elongation at break of the elastomer permit to withstand the stretch and abrasion due to forces encountered in object manipulation and exploration [40].

\subsection{Mechanotransduction core Technology}

Several types of artificial sensitive skin have been proposed and a large number of transduction mechanisms have been reported in the literature. Mechanoelectrotransduction (or mechanoneurotransduction) occurs when an external stimulus transfers energy to the human finger pad, in contact mode (e.g. mechanical probing) or contactless (e.g. heat transfer via radiation), to elicit sequences of electrical discharges that reach the brain via the afferent pathways and code the stimulus in a perceptual form.

Several reviews of tactile sensors have been proposed with respect to synthetic approaches for the design of the skin and transduction mechanisms [10, 41-46], and novel trends consider the integration of cell-based living structures [47-49]. Like sense of touch in humans, it is desirable to have tactile arrays, or a coordinated group of distributed tactile sensors, with density and spatial distribution of taxels (tactile elements) depending on the body site where the sensors are installed [50]. For the sites like fingertips, that are involved in tasks like exploration and fine manipulation, a large number of quick responding (response time of the order of few milliseconds) taxels are needed in a small space (with spatial resolution of about $1 \mathrm{~mm}$ ). Such spatial constraints can be met by miniaturizing the sensors and placing them over large areas in an array like fashion. Common synthetic tactile transduction techniques are based on capacitive, piezoelectric, piezoresistive, inductive, optoelectric and strain gauge methods. The main characteristics associated with these techniques are briefly illustrated hereafter.

Capacitive sensors consist of two conductive plates with a dielectric material sandwiched between them. For parallel plates, the capacitance value is expressed as $C=\left(A \varepsilon_{0} \varepsilon_{r}\right) / d$, where $C$ is the capacitance, $A$ is the area of the two plates, $\varepsilon_{0}$ is the permittivity of free space, $\varepsilon_{r}$ is the relative permittivity of the dielectric material and $d$ is the distance between the plates [35, 51-56]. Novel sensor concepts recently being proposed include the capacitive sensors with soft encapsulation materials and textile-based conductive electrodes [57].

Piezoelectric sensors are composed of piezoelectric materials, such as poled ceramic lead zirconium titanate (PZT) and polyvinylidene fluoride (PVDF), which respond to the applied force/pressure [58-64]. Piezoelectric sensors are often named as ultrasonic, when using elements that emit an ultrasonic pulse within a medium (a rubber pad); the pulse is propagated through the medium and reflected. This echo pulse is then received by the emitting source. The transit time of the pulse is proportional to the thickness of the medium, 
therefore the strength of the echo pulse depends on acoustic properties of the medium and on the tactile stimulation conditions [65].

Piezoresistive sensors consist of a pressure sensitive element which changes its resistance upon applied force. The resistance value of a resistor having length $l$ and cross-sectional area $A$ is given by: $R=\rho(l / A)$. According to this relationship, the resistance value is determined by both bulk resistivity $\rho$ and dimensions, with dominant variational effects associated mainly to resistivity changes [66-69].

Strain gauge sensors consist of a resistive elastic unit, whose change in resistance is a function of the applied strain as shown by the equation $d R / R=G \varepsilon$, where $R$ is the resistance, $\varepsilon$ is the strain and $G$ the gauge factor. If compared to piezoresistive sensors, the mechanotransduction properties of strain gauge devices are dominated by form factors rather than by variations in resistivity [70-73].

Inductive sensors are composed of a primary coil which induces a magnetic field sensed in a secondary sense coil; the mutual inductance between the coils modulates as a consequence of the applied load, thus modulating the amplitude and phase of the voltage measured in the sense coil $[74,75]$.

Optoelectric sensors employ a light source, a modulatory medium, a transmission medium and a photodetector, the latter often in the form of camera or photodiode. Transduction occurs when changes in the tactile medium modulate transmission intensity or the spectrum of the source light, as a consequence of variations in the applied force [76-80].

Table 1. Fully-synthetic tactile sensing: transduction methods, advantages and disadvantages (from [43-45, 47, 81]).

\begin{tabular}{|c|c|c|c|}
\hline $\begin{array}{l}\text { TRANSDUCTION } \\
\text { METHOD }\end{array}$ & $\begin{array}{l}\text { MODULATED } \\
\text { PARAMETER }\end{array}$ & ADVANTAGES & DISADVANTAGES \\
\hline Capacitive & Change in capacitance & $\begin{array}{ll}\text { - } & \text { High spatial resolution } \\
\text { - } & \text { Good frequency response } \\
\text { - } & \text { Long term drift stability } \\
- & \text { High sensitivity } \\
- & \text { Low temperature sensitivity } \\
- & \text { Low power consumption }\end{array}$ & $\begin{array}{ll}\text { - } & \text { Severe hysteresis } \\
\text { - } & \text { Stray capacitance } \\
\text { - } & \text { Complex electronics } \\
\text { - } & \text { Noise susceptible }\end{array}$ \\
\hline Piezoelectric & Strain (stress) polarization & $\begin{array}{ll}- & \text { Flexibility } \\
- & \text { Workability } \\
- & \text { Chemical stability } \\
\text { - } & \text { Good high-frequency response }\end{array}$ & $\begin{array}{ll}- & \text { High temperature sensitivity } \\
\text { - } & \text { Poor spatial resolution } \\
\text { - } & \text { Dynamic sensing only } \\
\text { - } & \text { Simple electronics }\end{array}$ \\
\hline Piezoresistive & Change in resistance & $\begin{array}{ll}- & \text { Flexibility } \\
- & \text { High spatial resolution } \\
- & \text { Good sensitivity } \\
- & \text { Low noise } \\
- & \text { Low cost } \\
- & \text { Simple electronics }\end{array}$ & $\begin{array}{ll}- & \text { Large hysteresis } \\
- & \text { Low frequency response } \\
- & \text { Low repeatability }\end{array}$ \\
\hline Strain gauge & Change in resistance & $\begin{array}{ll}- & \text { Sensing range } \\
- & \text { High sensitivity } \\
- & \text { Low cost }\end{array}$ & $\begin{array}{ll}\text { - } & \text { High hysteresis } \\
\text { - } & \text { Non-linear response } \\
\text { - } & \text { Susceptible to temperature } \\
\text { changes } \\
\text { - }\end{array}$ \\
\hline Inductive & Change in inductance & $\begin{array}{ll}- & \text { High sensitivity } \\
- & \text { High dynamic range } \\
\text { - } & \text { No mechanical hysteresis }\end{array}$ & $\begin{array}{lll}- & \text { Usage limited } & \text { to } \\
\text { nonmagnetic mediums } & \\
\text { - } & \text { Low spatial resolution } & \end{array}$ \\
\hline
\end{tabular}




\begin{tabular}{|l|l|l|l|}
\hline & & - Linear response & - Low repeatability \\
& & - Physical robustness & - Complex electronics \\
\hline & & - High density & - Large size \\
Optoelectric & $\begin{array}{l}\text { Light intensity/spectrum } \\
\text { change }\end{array}$ & - High spatial resolution & - Rigidness \\
& & Capability to sense both shear and & $-\begin{array}{l}\text { Loss of light by } \\
\text { microbending, causing } \\
\text { distortion of signal }\end{array}$ \\
\hline
\end{tabular}

\subsection{Neuromorphic representation of tactile information for biomimetic tactile computation}

In order to develop biomimetic tactile sensors, the computational characteristics of mechanoreceptors should be reproduced in addition to their physical features, and biomimetic sensors shall convey the same information about external stimuli as the biological sensors. The accuracy of the reproduction of biological information processing is not straightforward to test, since we can only study a limited set of stimuli, both with natural and artificial sensors. There are two options then. The first is to build ad hoc sensors to reproduce some selected aspects of tactile processing, as for instance sharp edge detection or stimulus intensity. The other is to build biomimetic sensors capturing the basic rules of peripheral tactile computation to reproduce the flexibility of natural sensors. As a matter of fact, in humans the long distance signalling between the periphery and the central nervous system occurs via a temporal sequence of neural spikes flowing in parallel along the afferent pathways, i.e. the communication can be considered as asynchronousdigital per each receptor channel (see Section 2). Along this direction, an emerging engineering trend is neuromorphic mechanotransduction, i.e. addressing bio-mimicry in terms of the used tactile code [27]. With neuromorphic signalling no information is therefore associated to the amplitude of the output signals of each taxel, whereas the encoding of tactile experience emerges from the temporal modulation of the (on-off) neural spiking behaviour of artificial receptors and from the spatial map of active taxels.

In order to achieve a better information processing performance reproducing the temporal spike modulations of biological neurons a series of step can be taken, as illustrated in Figure 3 and explained in the following.

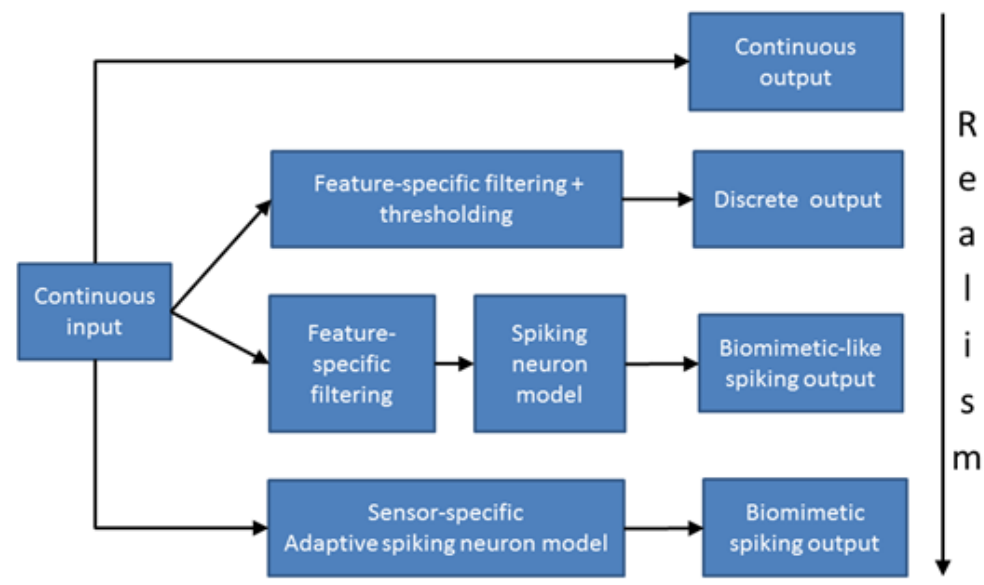

Figure 3. Possible approaches for soft-neuromorphic tactile sensing. Middle column shows the processing applied to the cntinouous output of sensors to obtain the output in the right column. Outputs are arranged from top to bottom in order of increasing realism, i.e., increasing similarity to biological outputs. 
The starting condition, which is not neuromorphic at all but can still be sufficient to convey basic information, is to leave the sensors output unprocessed except for noise-filtering and renormalization. The first step to achieve a biomimetic information representation is to convert the continuous output of the sensor arrays into discrete voltage pulses similar to (population) spikes. Besides making the signal closer to real mechanoreceptors output, this operation drastically reduces the size of the signal at the price of small or no information loss. A signal discretization can be achieved with simple signal processing tools, and computing spike times with a biomimetic approach, i.e., by means of a neuronal model reproducing the main features of the neurons biophysics, has a series of advantages. Crucially, such models can nowadays be implemented in hardware [82] and ensure high performance even in the case of unexpected stimuli. Moreover, such an approach will be easier to translate into brain-machine interface applications such as upper limb prostheses since it is reasonable to expect that decoding sensors inputs injected into the nervous system will be easier and faster to learn if these inputs are similar to the natural spike trains emitted by mechanoreceptors. Therefore the neuromorphic approach creates the ground for a natural integration between the artificial sensing system and the natural neural afferent pathways [83], and can represent a further advancement of approaches targeting the restoration of a missing sense of touch via neuroprostheses [84].

In Section Error! Reference source not found. we have seen how tactile sensory information is processed by different kind of mechanoreceptors (Figure 2). To take into account this variety of processing mechanisms a possible biomimetic approach is to build a parallel layer of computation with different processors reproducing the specific behaviours of the different mechanoreceptors [85, 86]. At a first stage, a single reference neuronal model can be adopted and specific features of the different neuron kinds can be achieved by standard non-biomimetic signal processing applied to the voltage input. This approach has been for instance used by Bensmaia and colleagues [87]. The final aim however should be to achieve the same pattern dynamics with biologically sound neuronal models. In this perspective, simple models like the leaky-integrate and fire neuron [88] should be discarded since they fail to reproduce the adaptation mechanisms that are the very core of the different mechanoreceptors behaviours [89]. On the other hand models such as the Hodgkin and Huxley neuron are computationally expensive since they aim at reproducing the details of the active channels dynamics [89], which does not currently seem to be relevant for this stage of information processing. Appropriate models to reproduce the mechanoreceptors are the Izikevich neuron model [90], and the Adaptive Exponential Integrate and Fire neuron model [91], both capturing at the same time adaptive mechanisms and the main features of the action potential shape. Which one of the two is more suited depends on factors such as the hardware implementation or the complexity of the computation.

In Section Error! Reference source not found. we also highlighted the key role played by the temporal structure of the firing (rather than the total amount of spikes fired) in tactile information processing. It is then crucial to reproduce the main timescales present in mechanoreceptors in the engineered model: as we have seen the adaptation onset and recovery timescale is probably the most important but also membrane timescale, synaptic opening and closing times, and, as shown in Figure 2, the synaptic delays should be taken into account, at least implicitly. Finally, we would like to stress that reproducing the temporal structure of the mechanoreceptors firing does not mean to reproduce the precise time of each spike down to arbitrary high temporal resolution. Every information-processing mechanism has an optimal timescale that can be estimated in different ways [92-94]. Timescales faster than the optimal one might then be not discriminated by downstream layers of information processing, because of biophysical constraints. Such a filtering plays also an important functional role since small jitters might be usually due to intrinsic noise. It is then of paramount importance to estimate the optimal timescale for peripheral tactile processing also to set the indicative 
temporal resolution at which we must aim to reproduce mechanoreceptors spike patterns.

\section{CASE STUDY TACTILE SENSING TECHNOLOGY: THE POSFET DEVICE}

The POSFET (Piezoelectric Oxide Semiconductor Field Effect Transistor) devices based tactile sensing arrays presented in this section have been designed for spatio-temporal parameters similar to that of cutaneous sensing at human fingertip. As described later in this section, the POSFET devices on the tactile sensing chips are realized by depositing a piezoelectric polymer, PVDF-TrFE (Polyvinylidene Fluoride Trifluoroethylene), on the gate area of a MOS transistor [95-99]. The resulting marriage of transducer and electronics is advantageous in many ways. In addition to minimizing the utilization of space, the marriage of transducer and electronics results in better force resolution, spatial resolution and signal to noise ratio.

\subsection{Structure and Working of a POSFET device}

The structure of POSFET touch sensing device, shown in Figure 4 (inset)Error! Reference source not found., is similar to a metal-ferroelectric-metal-insulator-semiconductor FeRAM (Ferroelectric Random Access Memory). The remanent polarization $\left(P_{r}\right)$, of the piezoelectric polymer film, and the charge neutrality condition, result in the appearance of fixed charges $\pm Q$ on the surface of polymer. This results in accumulation of charge carriers at the surface of the semiconductor - according to the polarization direction. When piezoelectric polymer is polarized along thickness and the mechanical stress is also applied along this direction, the mechanical stress $T_{3}$, electric field $E_{3}$ and electric displacement $D_{3}$ are related as [100-102]:

$$
D_{3}=d_{33} T_{3}+\varepsilon_{33} E_{3}
$$

Where, $d_{33}$ and $\varepsilon_{33}$ are the piezoelectric and dielectric constants of piezoelectric polymer respectively. Following (1), the electric displacement and hence the polarization can be controlled by the electric field $E_{3}$ and the applied force $F$ or stress $T_{3}$. While former is used in FeRAM to switch the polarization state, the latter is used in the POSFET touch sensing devices to modulate the charge in induced channel of underlying MOS device [4]. Thus, the contact force variation is directly reflected into channel current of POSFET devices -

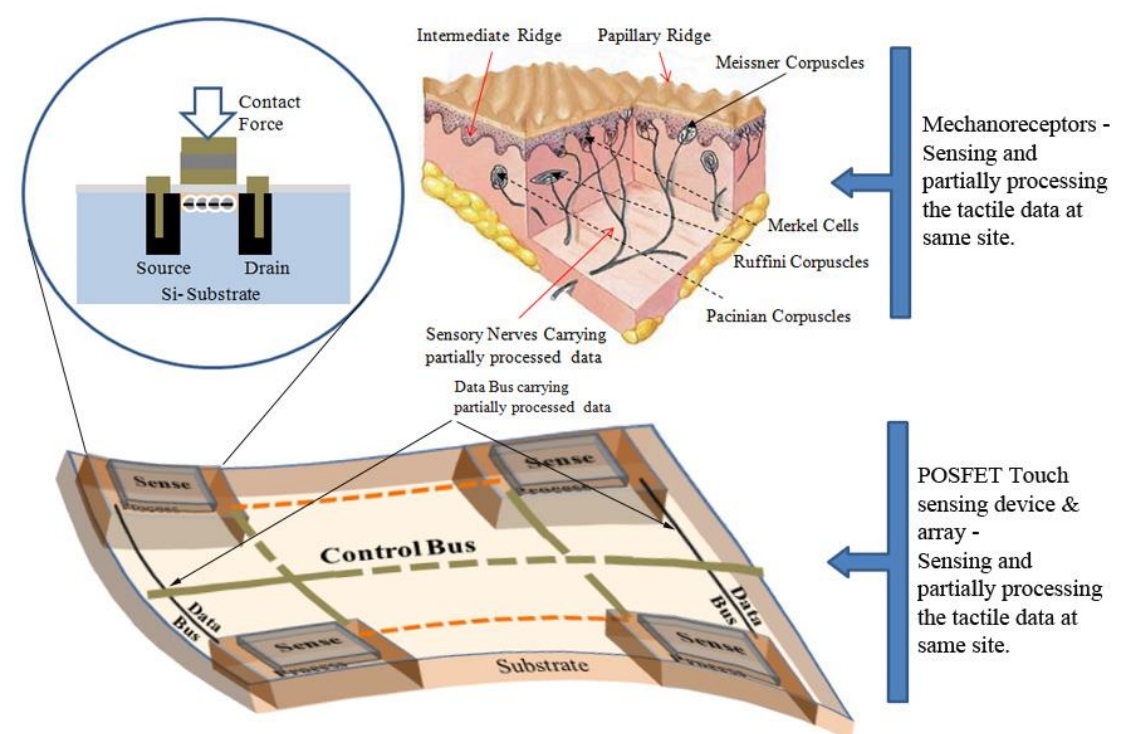

Figure 4. The functional comparison of a POSFET touch sensing device with the mechanoreceptors in the skin. 
which can be amplified by POSFET itself and further processed by the electronic circuitry that may be present on the same chip. Thus, each touch sensing element or taxel is an integral "sensotronic" unit, comprising of transducer and the transistor, which is capable of 'sensing and partially processing at same site'. In this context, a POSFET touch sensing device can be compared with the mechanoreceptors in human skin - that not only sense the contact parameters, but also partially process the tactile data at same site [22]. This analogy, between POSFET touch sensing device and the mechanoreceptors in the skin, is shown in Error! Reference source not found.4. Another feature of POSFET touch sensing devices is their ability to detect contact temperature variations. With a proper arrangement, this feature can be turned into an advantage and the POSFET device can be made multifunctional - capable of detecting both dynamic contact forces and the contact temperature.

A similar approach, utilizing extended gates, has also been adopted for ultrasonic [103] and force sensing [104]. The extended gate approach brings the sensor and conditioning electronics closer and hence the overall response is better than the conventional approach - where the sensor and conditioning electronics are placed apart. Overall, the sensitivity of POSFET based tactile sensing devices is higher than that of extended gates based tactile sensors. The presence of polymer only on the gate area, as in POSFET devices, results in a lesser polymer area (compared to that of an extended gate based tactile sensor). This means, for same thickness of the polymer, the effective capacitance is lesser and so is the $R C$ time constant. The large interconnects needed for the extended gate are also absent in case of POSFET - which further reduces the $R C$ time constant. One of the direct fallouts of reduced $R C$ constant is the faster response and improved range of frequencies. Besides this the absence of extended gates frees some of the real estate on the silicon wafer, which can be used for onchip electronics as done in the recent version of POSFETs [96, 97, 99]. The reliable interconnects between extended gate and MOS transistor is also an issue - more so in case of flexible touch sensing devices.

\subsection{POSFET Tactile Arrays - Design, Fabrication and Evaluation}

As discussed in previous sections, the human sense of touch like spatio-temporal response can be obtained by miniaturizing the sensors and then placing them over large area. The 5x5 POSFET tactile sensing arrays, with partly shown in Figure (a), have been designed to have spatial resolution and acuity similar to that of human fingertips. The overall dimension of the initial version of this tactile sensing array was $1.5 \mathrm{~cm} \times 1.5$ $\mathrm{cm}$, with each POSFET touch sensing device on the array designed to be $1 \mathrm{~mm} \times 1 \mathrm{~mm}$ in size - ensuring human like spatial acuity. The center-center distance of $1.5 \mathrm{~mm}$ between two adjacent taxels in the initial



(a)

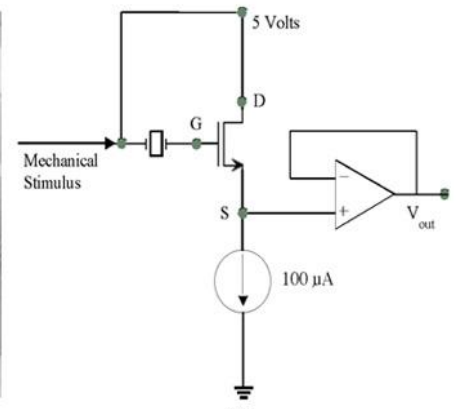

(b)

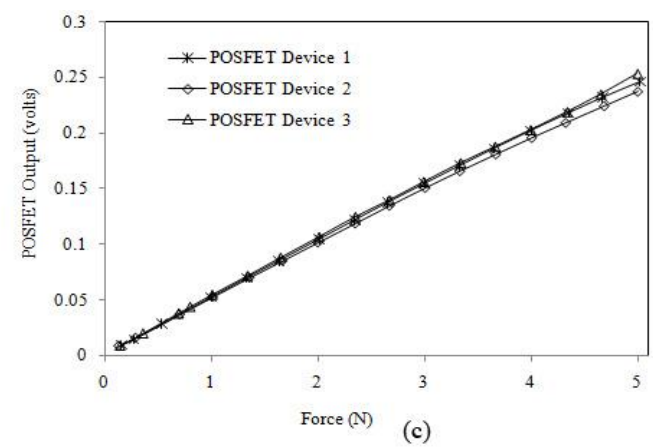

(c)

Figure 5. (a) The SEM picture of a part of 5x5 POSFET tactile sensing array [1]. The device dimension and distance between two adjacent devices are clearly marked. (b) The source-follower arrangement, with floating gate, used to evaluate the performance of a POSFET touch sensing device. (c) Response of three POSFET touch sensing devices when a $20 \mathrm{~Hz}$ sinusoidal force is applied on top in normal direction [4]. 
version of POSFET chips ensured human like spatial resolution. The spatial resolution of about $1 \mathrm{~mm}$ possible with POSFET based devices can be further improved by reducing the spacing between POSFET devices and also by reducing the size of the devices. The most recent version of POSFET chips have reduced number of taxels, i.e. $4 \times 4$, for optimal use of interconnects when sensors are read in row-column fashion [96, 99]. The overall size of the chip is $10.1 \mathrm{~mm} \times 8.1 \mathrm{~mm}$, with $1 \mathrm{~mm}$ center-to-center pitch of sensor elements. Each POSFET in the most recent version has been designed to have an active area of $0.9 \mathrm{~mm} \times 0.6 \mathrm{~mm}$.

The fabrication steps for POSFET tactile sensing arrays can be divided into two parts: a) developing MOS device, and b) depositing the piezoelectric polymer [105]. The MOS part of the POSFET touch sensing devices is obtained by using the n-MOS technological module of a non-standard CMOS technology based on $4 \mu \mathrm{m}$ p-well ion sensitive FET (ISFET)/CMOS process. A $\mathrm{Si}_{3} \mathrm{~N}_{4} / \mathrm{SiO}_{2}$ double layer is used as a gate dielectric. The piezoelectric polymer related steps include spin coating of polymer film; annealing; top metallization; dry etching and poling of polymer films. For a uniform response among various sensors, it is desired to have the polymer film with uniform thickness and hence the deposition method must be carefully chosen. The thickness of polymer film depends on the solution concentration, spinner's speed and time of spinning. As an example, a $2.5 \mu \mathrm{m}$ thick PVDF-TrFE polymer film can be deposited by spin coating a $10 \%$ solution at 3000 rpm for 30 seconds [106]. For the same concentration, the thickness of polymer film will decrease with increase in spinner's speed or increase in spinning time. Similarly, a higher concentration solution and higher spinning speed can be used to obtain $2.5 \mu \mathrm{m}$ thick films. After deposition, the polymer films are annealed at $120^{\circ} \mathrm{C}$ for three hours to enhance the crystallization, to evaporate any left out solvent and to remove any local stress generated during deposition. After this, the piezoelectric film is polarized to introduce the piezoelectric properties. An electric filed strength of 80-100 V/ $\mu \mathrm{m}$ is required to polarize the PVDF-TrFE films. The details of fabrication of most recent POSFETs are given in [96, 99].

To evaluate the performance of a POSFET touch sensing device a dynamic normal force is applied on the top metal and the change in channel current is observed. The POSFET device is connected in a source-follower circuit arrangement with floating gate, as shown in Figure 5(b). The source-follower configuration results in less than unity gain, and hence POSFET devices can be tested for wide range of forces. Alternately, common
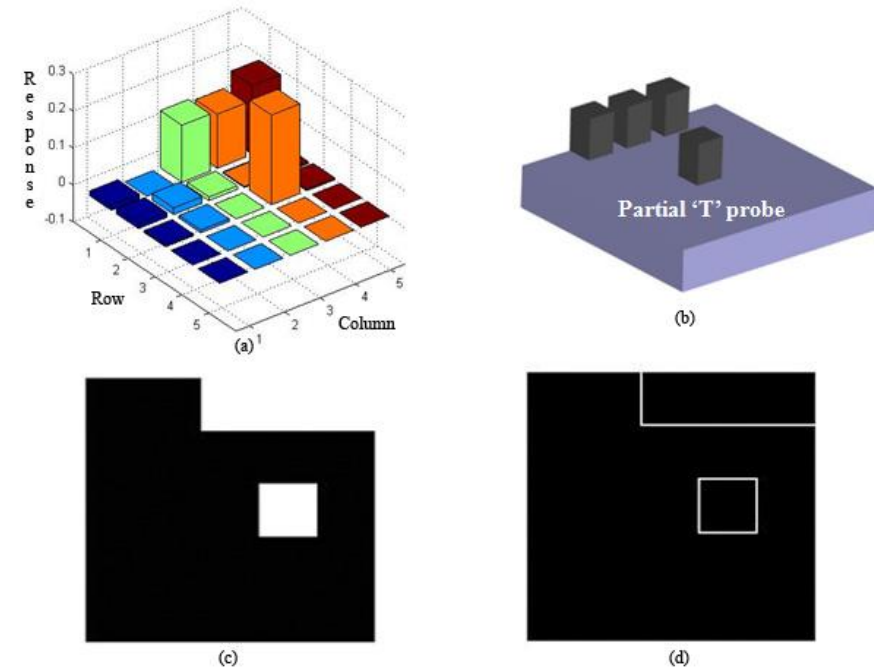

Figure 6. (a) Response of various POSFET devices on the array when they are simultaneously pressed with the probe shown in (b); (b) The partial "T" shaped probe; (c) Binary image of the response in (a); (d) Edge detection using the response data of various POSFET devices on the array. 
source configuration can be used to have better force resolution. The response of POSFET touch sensing device is linear, shown in Figure (c), over tested range of dynamic normal forces (0.15-5N, sinusoidal $20 \mathrm{~Hz})$. The output of POSFET devices over the tested range is linear with $49 \mathrm{mV} / \mathrm{N}$ average slope. The output of the most recent version of POSFET devices is linear with $102 \mathrm{mV} / \mathrm{N}$ average slope [96, 99]. The POSFET devices were tested only up to $2.13 \mathrm{kHz}$ as this frequency is already higher than that perceived by human fingertip. In principle, the POSFETs will have much higher bandwidth due to the higher bandwidth of silicon based MOS devices and the high pass characteristics of piezoelectric polymers.

The simultaneous excitation of many sensing elements (i.e. application of mechanical stimulus on multiple POSFET elements) is another important test to measure the spatial resolution and the object imaging capability of a tactile sensing array. A snap-shot of the response of various POSFET tactile sensors when normal force ( $670 \mathrm{~Hz}$ sinusoidal) is applied on the array with the partial ' $\mathrm{T}$ ' shaped probe, is given in Figure (a). The scheme of the probe used for this purpose is also shown in Figure 6(b). The variation among the responses of the taxels that are pressed is small. A snap-shot of the binary images, obtained from the normalized response of various taxels, is also shown in Figure 6(c). Similar results are obtained by varying the frequency of applied sinusoidal normal forces. The negligible cross-talk confirms that the POSFET tactile sensing arrays have the spatial resolution $(1 \mathrm{~mm})$ for which they are designed. The data obtained from these experiments can also be used to detect the edges, as shown in Figure 6(d). While the dynamic stimulus in above results varies with time, it is stationary in space. A real world stimulus may vary both in time and space. When such a stimulus (a ring shaped probe rolled over the diagonal elements of the array) was applied, the POSFETs yielded good results [1] - thus demonstrating their capability to detect stimuli that varies both in time and space. The POSFETS are able to meet many features of human fingertips and they are compared in Table 2. Recent development such as flexible or bendable chips [107-109], printing of sensors [110, 111] etc. offer new avenues for cost-effective bio-inspired hardware allowing skin sensor hardware to conform to the $3 \mathrm{D}$ curved surfaces.

Table 2. The comparison of the tested features of POSFET tactile sensing arrays with the cutaneous/tactile sense of human fingertips.

\begin{tabular}{|l|l|l|}
\hline Feature & Human Fingertips & POSFET Array \\
\hline Mechanoreceptor Density & $241 / \mathrm{cm}^{2}$ & $25 / \mathrm{cm}^{2}$ \\
\hline Multifunctionality & Yes & Yes \\
\hline Typical Range of Forces during normal manipulative tasks & $0.1-0.9 \mathrm{~N}$ & $0.15-5 \mathrm{~N}$ (tested) \\
\hline Detectable Frequency range of vibration & DC-700 Hz & $2-2130 \mathrm{~Hz}$ (tested) \\
\hline Spatial Resolution & $<1 \mathrm{~mm}$ & $-1 \mathrm{~mm}$ \\
\hline Receptor level processing of contact data & Yes & Yes \\
\hline
\end{tabular}

\section{OTHER EXAMPLES OF BIO-INSPIRED TACTILE SENSING}

There are many studies aiming to realize biologically inspired tactile sensors. These include the neural spike processing based biologically inspired tactile sensor array reported in [112]. These tactile sensors array integrate the asynchronous data processing on-chip with the sensor array and convert the high-bandwidth raw input data into higher-level information with lower bandwidth requirements. As opposed to traditional rate based neural spike codes, these tactile sensor arrays use phase-based computation primitives and are able to compute in real-time the point-of-contact, force magnitude, force direction, and the presence or absence of slips etc. Another example of bio-inspired tactile sensing, reported in [113], embeds many receptors randomly in soft material so as to provide different kinds of sensing modalities. Based on this design principle an anthropomorphic fingertip is also developed. 
Another example of biomimetic tactile sensor array reported in [114] mimics the mechanical properties and distributed touch receptors of the human fingertip. Modelled after the human digit, the tactile sensor consists of a rigid central core surrounded by a weakly conductive fluid contained within a silicone elastomeric skin. The sensor uses the deformable properties of the finger pad as part of the transduction process. Multiple electrodes mounted on the surface of the rigid core and connected to impedance-measuring circuitry embedded within the core measure the contacts force. Basically, the external contact forces deform the fluid path around the electrodes, which results in a distributed pattern of impedance changes containing information about those forces and the objects that applied them. As with biological fingertips the sensor incorporates the low-pass filter effects of cosmetic, protective skin and fluid into the transduction process.

\section{CONCLUSION}

The ways in which biological systems gather and process information may not always lead to the best engineering solutions. Nevertheless, they provide useful insights into how behaving organisms respond to dynamically changing environments and hence provide a comprehensive multilevel conceptual framework within which to organize the overall task. Thus, rather than imitating, understanding the working of a biological system is more important. Rather than implementing dedicated algorithms to extract specific features, a neuromorphic computational architecture allows to achieve the desired functionality via the implemented structure. Keeping this in view, some of the design cues inspired from human tactile sensing system have been presented in this chapter and used as desiderata for designing the bio-inspired tactile sensing arrays. Some of these cues (structural or functionality) have been used in the examples of bio-inspired tactile sensors presented in this chapter. 


\section{REFERENCES}

[1] R. S. Dahiya, L. Lorenzelli, G. Metta, and M. Valle, "POSFET Devices Based Tactile Sensing Arrays," in IEEE-ISCAS 2010, The IEEE International Symposium on Circuits and Systems, Paris, France, 2010, pp. 1-4.

[2] R. S. Dahiya, G. Metta, M. Valle, and G. Sandini, "Tactile Sensing: From Humans to Humanoids," IEEE Transactions on Robotics, vol. 26, pp. 1-20, 2010.

[3] G. Westling and R. S. Johannson, "Factors influencing the force control during precision grip," Experimental Brain Research, vol. 53, pp. 277-284, 1984.

[4] R. S. Dahiya, G. Metta, M. Valle, L. Lorenzelli, and A. Adami, "Piezoelectric Oxide Semiconductor Field Effect Transistor Touch Sensing Devices," Applied Physics Letters, vol. 95, pp. 034105 (1-3), 2009.

[5] M. Pare, C. Behets, and O. Cornu, "Paucity of presumptive Ruffini corpuscles in the index finger pad of humans," The Journal of Comparative Neurology, vol. 456, pp. 260-266, 2003.

[6] V. B. Mountcastle, The Sensory Hand: Neural Mechanisms of Somatic Sensation. Cambridge, Massachusetts and London, England: Harvard University Press, 2005.

[7] R. S. Fearing, "Tactile Sensing Mechanisms," The International Journal of Robotics Research, vol. 9, pp. 3-23, 1990.

[8] R. D. Howe, "Tactile Sensing and Control of Robotics Manipulation," Journal of Advanced Robotics, vol. 8, pp. 245-261, 1994.

[9] M. Shikida, T. Shimizu, K. Sato, and K. Itoigawa, "Active Tactile Sensor for Detecting Contact Force and Hardness of an Object," Sensors and Actuators A, vol. 103, pp. 231-218, 2003.

[10] M. H. Lee, H. R. Nicholls, "Tactile sensing for mechatronics - a state of the art survey," Mechatronics, vol. 9, pp. 1-31, 1999.

[11] R. S. Dahiya and M. Valle, Robotic Tactile Sensing - Technologies and System. Dordrecht: Springer, 2013.

[12] T. D. Crouch, Wings: a history of aviation from kites to the space age. New York: W.W. Norton \& Comapany, Inc., 2003.

[13] J. M. Wolfe, K. R. Kluender, D. M. Levi, L. M. Bartoshuk, R. S. Herz, R. L. Klatzky, and S. J. Lederman, Sensation and Perception. Sunderland, Massachusetts USA: Sinauer Associates Inc., 2006.

[14] D. Purves, G. J. Augustine, D. Fitzpatrick, W. C. Hall, A.-S. LaMantia, J. O. McNamara, and L. E. White, Neuroscience, 4th Ed. ed.: Sianuer Associates Inc., 2008.

[15] E. R. Kandel, J. H. Schwartz, and T. M. Jessell, Principles of Neural Science 4th ed.: McGraw-Hill Medical, 2000.

[16] J. M. Loomis, S. J. Lederman, Tactual Perception vol. 2 Cognitive Processes and Performances. N. York: John Wiley \& Sons, 1986.

[17] B. B. Edin and N. Johansson, "Skin strain patterns provide kinaesthetic information to the human central nervous system," The Journal of Physiology, vol. 487, pp. 243-251, 1995.

[18] V. E. Abraira and D. D. Ginty, "The Sensory Neurons of Touch," Neuron, vol. 79, pp. 618-639, Aug 212013.

[19] R. S. Johannson and A. B. Vallbo, "Tactile Sensibility in the Human Hand: Relative and Absolute Densities of Four Types of Mechanoreceptive Units in Glabrous Skin," The Journal of Physiology, vol. 286, pp. 283-300, 1979.

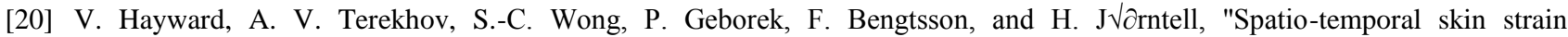
distributions evoke low variability spike responses in cuneate neurons," J. Royal Society Interface, vol. 11, April 6, 20142014.

[21] R. S. Johannson and I. Birznieks, "First Spikes in Ensembles of Human Tactile Afferents Code Complex Spatial Fingertip Events," Nature Neuroscience, vol. 7, pp. 170-177, 2004.

[22] R. S. Johannson and J. R. Flanagan, "Coding and use of tactile signals from the fingertips in object manipulation tasks," Nature Reviews Neuroscience, vol. 10, pp. 345-359, 2009.

[23] H. Jörntell, F. Bengtsson, P. Geborek, A. Spanne, A. V. Terekhov, and V. Hayward, "Segregation of Tactile Input Features in Neurons of the Cuneate Nucleus," Neuron, 2014.

[24] P. Dario, B. Hannaford, and A. Takanishi, "Guest Editorial Special Issue on Biorobotics," IEEE Trans. Robotics, vol. 24, pp. 34, 2008.

[25] R. Pfeifer, J. Bongard, and S. Grand, How the body shapes the way we think: a new view of intelligence: MIT press, 2007.

[26] C. Laschi, M. Cianchetti, "Soft robotics: new perspectives for robot bodyware and control," Bionics \&Biomimetics, vol. 2, pp 3, 2014.

[27] G. Spigler, C. M. Oddo, and M. C. Carrozza, "Soft-neuromorphic artificial touch for applications in neuro-robotics," in 4th IEEE RAS \& EMBS International Conference on Biomedical Robotics and Biomechatronics (BioRob), 2012, pp. $1913-1918$.

[28] J. Scheibert, S. Leurent, A. Prevost, and G. Debregeas, "The Role of Fingerprints in the Coding of Tactile Information Probed with a Biomimetic Sensor," Science, vol. 323, pp. 1503-1506, 2009.

[29] R. Candelier, A. Prevost, and G. Debrégeas, "The role of exploratory conditions in bio-Inspired tactile sensing of single topogical features," Sensors, vol. 11, pp. 7934-7953, 2011.

[30] G. Vasarhelyi, M. Adam, E. Vazsonyi, I. Barsony, and C. Ducso, "Effects of the elastic cover on tactile sensor arrays," Sens. 
Actuat. A-Phys., vol. 132, pp. 245-251, Nov 82006.

[31] C. M. Oddo, L. Beccai, J. Wessberg, H. B. Wasling, F. Mattioli, and M. C. Carrozza, "Roughness encoding in human and biomimetic artificial touch: spatiotemporal frequency modulation and structural anisotropy of fingerprints," Sensors, vol. 11, pp. 5596-5615, 2011.

[32] J. J. Cabibihan, M. C. Carrozza, P. Dario, S. Pattofatto, M. Jomaa, and A. Benallal, "The Uncanny Valley and the Search for Human Skin-Like Materials for a Prosthetic Fingertip," in 6th IEEE-RAS Int. Conf. on Humanoid Robots, 2006, pp. $474-477$.

[33] M. Controzzi, M. D'Alonzo, C. Peccia, C. M. Oddo, M. C. Carrozza, and C. Cipriani, "Bioinspired fingertip for anthropomorphic robotic hands," Applied Bionics and Biomechanics, vol. 11, pp. 25-38, 2014.

[34] D. J. Montana, "Contact stability for two-fingered grasps," IEEE T. Robotic. Autom., vol. 8, pp. 421-430, 1992.

[35] N. Wettels, V. J. Santos, R. S. Johansson, G. E. Loeb, "Biomimetic Tactile Sensor Array," Adv. Robot., vol. 22, pp. 829-849, 2008.

[36] H. Okada, M. Yawata, M. Ishida, K. Sawada, and H. Takao, "A membrane type Si-MEMS tactile imager with fingerprint structure for realization of slip sensing capability," in 23rd IEEE Int. Conf. on MicroElectroMechanical Systems (MEMS), 2010, pp. 608-611.

[37] R. S. Dahiya, M. Gori, "Probing With and Into Fingerprints," J. Neurophysiology, vol. 104, pp. 1-3, 2010.

[38] K. B. Shimoga and A. A. Goldenberg, "Soft materials for robotic fingers," in IEEE Int. Conf. on Robotics \& Automation, pp. 1300-1305, 1992.

[39] G. Vasarhelyi, B. Fodor, and T. Roska, "Tactile sensing-processing: Interface-cover geometry and the inverse-elastic problem," Sens. Actuat. A-Phys., vol. 140, pp. 8-18, Oct 12007.

[40] D. Roy, N. Wettels, G. E. Loeb, "Elastomeric skin selection for a fluid-filled artificial fingertip," J. Appl. Polym. Sci., pp. 1-10, 2012.

[41] D. D. Rossi, "Artificial tactile sensing and haptic perception," Measurement Science and Technology, vol. 2, p. $1003,1991$.

[42] V. Maheshwari and R. Saraf, "Tactile devices to sense touch on a par with a human finger," Angew. Chem. Int. Ed., vol. 47, pp. 7808-7826, 2008.

[43] R. S. Dahiya, G. Metta, M. Valle, and G. Sandini, "Tactile sensing - From humans to humanoids," IEEE Trans. Robot., vol. 26, pp. 1-20, 2010.

[44] H. Yousef, M. Boukallel, and K. Althoefer, "Tactile sensing for dexterous in-hand manipulation in robotics-A review," Sens. Actuat. A-Phys., vol. 167, pp. 171-187, 2011.

[45] M. I. Tiwana, S. J. Redmond, and N. H. Lovell, "A review of tactile sensing technologies with applications in biomedical engineering," Sens. Actuat. A-Phys., vol. 179, pp. 17-31, 2012.

[46] M. L. Hammock, A. Chortos, B. C. Ä. Tee, J. B. Ä. Tok, and Z. Bao, "25th Anniversary Article: The Evolution of Electronic Skin (E,ÄêSkin): A Brief History, Design Considerations, and Recent Progress," Adv. Materials, vol. 25, pp. 5997-6038, 2013.

[47] C. Lucarotti, C. M. Oddo, N. Vitiello, and M. C. Carrozza, "Synthetic and bio-artificial tactile sensing: A review," Sensors, vol. 13, pp. 1435-1466, 2013.

[48] A. Adamatzky, "Slime mould tactile sensor," Sensors and Actuators B: Chemical, vol. 188, pp. 38-44, 2013.

[49] D. Cheneler, J. Bowen, and G. Kaklamani, "Transient bioimpedance monitoring of mechanotransduction in artificial tissue during indentation," Journal of Electrical Bioimpedance, vol. 5, pp. 55-73, 2014.

[50] R. S. Dahiya, G. Metta, M. Valle, and G. Sandini, "Tactile Sensing - From Humans to Humanoids," IEEE Transactions on Robotics, vol. 26, pp. 1-20, 2010.

[51] M. Y. Cheng, X. H. Huang, C. W. Ma, and Y. J. Yang, "A flexible capacitive tactile sensing array with floating electrodes," J. Micromech. Microeng., vol. 19, p. 115001, 2009.

[52] H. B. Muhammad, C. M. Oddo, L. Beccai, C. Recchiuto, C. J. Anthony, M. J. Adams, M. C. Carrozza, D. W. L. Hukins, and M. C. L. Ward, "Development of a bioinspired MEMS based capacitive tactile sensor for a robotic finger," Sens. Actuat. A-Phys., vol. 165, pp. 221-229, 2011.

[53] H. B. Muhammad, C. Recchiuto, C. M. Oddo, L. Beccai, C. J. Anthony, M. J. Adams, M. C. Carrozza, and M. C. L. Ward, "A capacitive tactile sensor array for surface texture discrimination," Microelectron. Eng., vol. 88, pp. 1811-1813, 2011.

[54] A. Medler, C. Patel, and J. Butcher, "A capacitive pressure sensor fabricated by a combination of SIMOX (SOI) substrates and novel etching techniques," J. Commun., vol. 47, pp. 6-8, 1996.

[55] S. T. Moe, K. Schiolberg-Henriksen, D. T. Wang, E. Lund, J. Nysaether, L. Furuberg, M. Visser, T. Fallet, and R. W. Bernstein, "Capacitive differential pressure sensor for harsh environments," Sens. Actuat. A-Phys., vol. 83, pp. 30-33, 2000.

[56] S. Renard, C. Pisella, J. Collet, F. Perruchot, C. Kergueris, P. Destrez, P. Rey, N. Delorme, and E. Dallard, "Miniature pressure acquisition microsystem for wireless in vivo measurements," IEEE-EMBS Special Topic Conference on Microtechnologies in Medicine \& Biology, pp. 175-179, 2002. 
[57] L. Viry, A. Levi, M. Totaro, A. Mondini, V. Mattoli, B. Mazzolai, and L. Beccai, "Flexible Three-Axial Force Sensor for Soft and Highly Sensitive Artificial Touch," Advanced Materials, vol. 26, pp. 2659-2664, 2014.

[58] G. M. Atkinson, R. E. Pearson, Z. Ounaies, C. Park, J. S. Harrison, and J. A. Midkiff, "Piezoelectric polyimide tactile sensors," in University/Government/Industry Microelectronics Symposium, 2003. Proceedings of the 15th Biennial, 2003, pp. 308-311.

[59] C. Cheng-Hsin, D. Wen-Bin, and L. Wen-Bin, "Flexible piezoelectric tactile sensor with structural electrodes array for shape recognition system," in Sensing Technology, 2008. ICST 2008. 3rd International Conference on, 2008, pp. 504-507.

[60] R. S. Dahiya, G. Metta, and M. Valle, "Development of fingertip tactile sensing chips for humanoid robots," in Mechatronics, 2009. ICM 2009. IEEE International Conference on, 2009, pp. 1-6.

[61] G. M. Krishna and K. Rajanna, "Tactile sensor based on piezoelectric resonance," IEEE Sens. J., vol. 4, pp. 691-697, Oct 2004.

[62] C. Y. Li, P. M. Wu, S. Lee, A. Gorton, M. J. Schulz, and C. H. Ahn, "Flexible dome and bump shape piezoelectric tactile sensors using PVDF-TrFE copolymer," J. Microelectromech. S., vol. 17, pp. 334-341, Apr 2008.

[63] K. Hosoda, Y. Tada, and M. Asada, "Anthropomorphic robotic soft fingertip with randomly distributed receptors," Robot. Auton. Syst., vol. 54, pp. 104-109, Feb 282006.

[64] R. Caliò, U. B. Rongala, D. Camboni, M. Milazzo, C. Stefanini, G. De Petris, and C. M. Oddo, "Piezoelectric Energy Harvesting Solutions," Sensors, vol. 14, pp. 4755-4790, 2014.

[65] S. Ando, "Ultrasonic Emission Tactile Sensing," IEEE Contr. Syst. Mag., vol. 15, pp. 61-69, Feb 1995.

[66] L. Beccai, S. Roccella, A. Arena, F. Valvo, P. Valdastri, A. Menciassi, M. C. Carrozza, P. Dario, "Design and fabrication of a hybrid silicon three-axial force sensor for biomechanical applications," Sens. Actuat. A-Phys., vol. 120, pp. 370-382, May 17 2005.

[67] L. Wang and D. J. Beebe, "A silicon-based shear force sensor: development and characterization," Sens. Actuat. A-Phys., vol. 84, pp. 33-44, Aug 12000.

[68] T. Mei, W. J. Li, Y. Ge, Y. Chen, L. Ni, and M. H. Chan, "An integrated MEMS three-dimensional tactile sensor with large force range," Sens. Actuat. A-Phys., vol. 80, pp. 155-162, Mar 102000.

[69] A. Tibrewala, A. Phataralaoha, and S. Buttgenbach, "Development, fabrication and characterization of a 3D tactile sensor," $J$. Micromech. Microeng., vol. 19, Dec 2009.

[70] J. C. Lee and D. W. Lee, "Flexible and tactile sensor based on a photosensitive polymer," Microelectron. Eng., vol. 87, pp. 1400-1403, May-Aug 2010.

[71] E. S. Hwang, J. H. Seo, and Y. J. Kim, "A polymer-based flexible tactile sensor for both normal and shear load detections and its application for robotics," J. Microelectromech. S., vol. 16, pp. 556-563, Jun 2007.

[72] H. J. Kwon and W. C. Choi, "Design and fabrication of a flexible three-axial tactile sensor array based on polyimide micromachining," Microsyst. Technol., vol. 16, pp. 2029-2035, Dec 2010.

[73] M. Tanaka, T. Iijima, Y. Tanahashi, and S. Chonan, "Development of a 3D tactile sensor," J. Mater. Process. Tech., vol. 181, pp. 286-290, Jan 12007.

[74] Y. Hasegawa, H. Sasaki, M. Shikida, K. Sato, and K. Itoigawa, "Magnetic actuation of a micro-diaphragm structure for an active tactile sensor," International Symposium on Micro-Nanomechatronics and Human Science, pp. 99-104, 2004.

[75] S. Takenawa, "A Magnetic type tactile sensor using a two-dimensional array of inductors," IEEE Int. Conf. Robot., pp. 15161521, 2009.

[76] S. Begej, "Planar and finger-shaped optical tactile sensors for robotic applications," IEEE Trans. Robot. Autom., vol. 4, pp. 472484 , Oct 1988.

[77] A. Persichetti, F. Vecchi, and M. C. Carrozza, "Optoelectronic-based flexible contact sensor for prosthetic hand application," Int. C. Rehab. Robot., pp. 415-420, 2007.

[78] R. Ahmadi, M. Packirisamy, J. Dargahi, and R. Cecere, "Discretely loaded beam-type optical fiber tactile sensor for tissue manipulation and palpation in minimally invasive robotic surgery," IEEE Sens. J., vol. 12, pp. 22-32, Jan 2012.

[79] V. Maheshwari, "High-Resolution Thin-Film Device to Sense Texture by Touch," Science, vol. 312, pp. 1501-1504, 2006.

[80] A. Levi, M. Piovanelli, S. Furlan, B. Mazzolai, and L. Beccai, "Soft, Transparent, Electronic Skin for Distributed and Multiple Pressure Sensing," Sensors, vol. 13, pp. 6578-6604, 2013.

[81] R. S. Dahiya and M. Valle, "Tactile Sensing for Robotic Applications," in Sensors, Focus on Tactile, Force and Stress Sensors, J. G. Rocha and S. Lanceros-Mendez, Eds., ed Vienna, Austria: InTech Open Access, 2008, pp. 289-304.

[82] S. Millner, A. Gr ${ }^{\circ}$ bl, K. Meier, J. Schemmel, and M.-O. Schwartz, "A VLSI implementation of the adaptive exponential integrate-and-fire neuron model," in Advances in neural information processing systems, 2010, pp. 1642-1650.

[83] E. Kim, K. Sugg, N. Langhals, S. Lightbody, M. Baltrusaitis, M. Urbanchek, P. Cederna, and G. J. Gerling, "An engineered tactile afferent modulation platform to elicit compound sensory nerve action potentials in response to force magnitude," in World Haptics Conference (WHC), 2013, 2013, pp. 241-246. 
[84] S. Raspopovic, M. Capogrosso, F. M. Petrini, M. Bonizzato, J. Rigosa, G. Di Pino, J. Carpaneto, M. Controzzi, T. Boretius, and E. Fernandez, "Restoring natural sensory feedback in real-time bidirectional hand prostheses," Science Translational Medicine, vol. 6, pp. 222ra19-222ra19, 2014.

[85] L. L. Bologna, J. r. m. Pinoteau, R. Brasselet, M. Maggiali, and A. Arleo, "Encoding/decoding of first and second order tactile afferents in a neurorobotic application," Journal of Physiology-Paris, vol. 105, pp. 25-35, 2011.

[86] L. Bologna, J. Pinoteau, J. Passot, J. Garrido, J. Vogel, E. R. Vidal, and A. Arleo, "A closed-loop neurobotic system for fine touch sensing," Journal of neural engineering, vol. 10, p. 046019, 2013.

[87] S. S. Kim, A. P. Sripati, S. J. Bensmaia, "Predicting the timing of spikes evoked by tactile stimulation of the hand," $J$. neurophysiology, vol. 104, pp. 1484-1496, 2010.

[88] N. Brunel, "Dynamics of neural networks," in Principles of Neural Coding, R. Q. Quiroga and S. Panzeri, Eds., ed Boca Raton: CRC Press, 2013.

[89] E. M. Izhikevich, "Which model to use for cortical spiking neurons?," IEEE trans.neural networks, vol. 15, pp. 1063-1070, 2004.

[90] E. M. Izhikevich, "Simple model of spiking neurons," IEEE Trans. Neural Networks, vol. 14, pp. 1569-1572, 2003.

[91] R. Brette and W. Gerstner, "Adaptive exponential integrate-and-fire model as an effective description of neuronal activity," $J$. neurophysiology, vol. 94, pp. 3637-3642, 2005.

[92] Y. Vázquez, E. Salinas, and R. Romo, "Transformation of the neural code for tactile detection from thalamus to cortex," Proc. National Academy of Sciences, vol. 110, pp. E2635-E2644, 2013.

[93] C. Kayser, N. K. Logothetis, and S. Panzeri, "Millisecond encoding precision of auditory cortex neurons," Proc. National Academy of Sciences, vol. 107, pp. 16976-16981, 2010.

[94] J. D. Victor and K. P. Purpura, "Spatial phase and the temporal structure of the response to gratings in V1," J. Neurophysiology, vol. 80, pp. 554-571, 1998.

[95] R. S. Dahiya, G. Metta, M. Valle, A. Adami, and L. Lorenzelli, "Piezoelectric oxide semiconductor field effect transistor touch sensing devices," Applied Physics Letters, vol. 95, pp. 034105-034105-3, 2009.

[96] R. S. Dahiya, A. Adami, C. Collini, and L. Lorenzelli, "POSFET Tactile Sensing Arrays using CMOS Technology," Sensors and Actuators - A, vol. 202, pp. 226-232, 2013.

[97] A. Adami, R. S. Dahiya, C. Collini, D. Cattin, and L. Lorenzelli, "POSFET touch sensor with CMOS integrated signal conditioning electronics," Sensors and Actuators A: Physical, vol. 98, pp. 502-507, 2012.

[98] R. S. Dahiya, D. Cattin, A. Adami, C. Collini, L. Barboni, M. Valle, L. Lorenzelli, R. Oboe, G. Metta, and F. Brunetti, "Towards Tactile Sensing System on Chip for Robotic Applications," IEEE Sensors Journal, vol. 11, pp. 3216-3226, 2011.

[99] R. S. Dahiya, A. Adami, L. Pinna, C. Collini, M. Valle, and L. Lorenzelli, "Tactile Sensing Chips With POSFET Array and Integrated Interface Electronics," IEEE Sensors Journal, vol. 14, pp. 3448-3457, 2014.

[100] R. S. Dahiya, M. Valle, R. Oboe, and D. Cattin, "Development and characterization of touch sensing devices for robotic applications," in IECON '09. 35th Annual Conference of IEEE Industrial Electronics. , Porto, Portugal, 2009, pp. 4245-4250.

[101] R. S. Dahiya, "Piezoelectric Tactile Sensors," in Wiley Encyclopedia of Electrical and Electronics Engineering, J. Webster, Ed., ed N. Jersey: John Wiley \& Sons, Inc., 2014.

[102] R. S. Dahiya, M. Valle, and L. Lorenzelli, "SPICE model for lossy piezoelectric polymers," IEEE Trans Ultrason Ferroelectr Freq Control, vol. 56, pp. 387-95, Feb 2009.

[103] R. G. Swartz and J. D. Plummer, "Integrated silicon-PVF2 acoustic transducer arrays," IEEE trans. Electron Devices, vol. 26, pp. 1920-32, 1979.

[104] I. Graz, M. kaltenbrunner, C. Keplinger, R. Schwodiauer, and S. Bauer, "Flexible ferroelectret field-effect transitor for largearea sensor skins and microphones," Applied Physics Letters, vol. 89, pp. 073501(1-3), 2006.

[105] R. S. Dahiya, M. Valle, G. Metta, L. Lorenzelli, and A. Adami, "Design and Fabrication of POSFET Devices for Tactile Sensing " in The 15th IEEE Int.Conf. on Solid-State Sensors, Actuators and Microsystems, Denver, USA, 2009, pp. 1881-1884.

[106] R. S. Dahiya, M. Valle, L. Lorenzelli, G. Metta, and S. Pedrotti, "Deposition Processing and Characterization of PVDF-TrFE Thin Films for Sensing Applications," in IEEE Sensors 2008, Lecce, Italy, 2008, pp. 490-493.

[107] R. S. Dahiya, A. Adami, C. Collini, L. Lorenzelli, "Bendable ultra-thin silicon chips on foil," in IEEE Sensors, 2012 , pp. 1-4.

[108] R. S. Dahiya and S. Gennaro, "Bendable Ultra-Thin Chips on Flexible Foils," IEEE Sensors J., vol. 13, pp. 4030-4037, 2013.

[109] R. S. Dahiya, A. Adami, C. Collini, and L. Lorenzelli, "Fabrication of Single Crystal Silicon Mirco-/Nanostructures and Transferring them to Flexible Substrates," Microelectronic Engineering, vol. 98, pp. 502-507, 2012.

[110] S. Khan, L. Lorenzelli, and R. S. Dahiya, "Bendable piezoresistive sensors by screen printing MWCNT/PDMS composites on flexible substrates," in 10th Conference on Ph.D. Research in Microelectronics and Electronics (PRIME), 2014, pp. 1-4.

[111] S. Khan, L. Lorenzelli, and R. S. Dahiya, "Screen printed flexible pressure sensors skin," in 25th Annual SEMI Advanced 
Semiconductor Manufacturing Conference (ASMC) 2014, pp. 219-224.

[112] A. Cassidy and V. Ekanayake, "A biologically inspired tactile sensor array utilizing phase-based computation," in IEEE Biomedical Circuits and Systems Conference (BioCAS) 2006, pp. 45-48.

[113] K. Hosoda, Y. Tada, and M. Asada, "Anthropomorphic robotic soft fingertip with randomly distributed receptors," Robotics and Autonomous Systems, vol. 54, pp. 104-109, 2006.

[114] N. Wettels, V. J. Santos, R. S. Johansson, G. E. Loeb, "Biomimetic Tactile Sensor Array," Adv. Robotics, vol. 22, pp. 829-849, 2008. 